Swarthmore College

Works

1995

\title{
Distinguishing Between Animates And Inanimates: Not By Motion
}

\section{Alone}

R. Gelman

Frank H. Durgin

Swarthmore College, fdurgin1@swarthmore.edu

L. Kaufman

Follow this and additional works at: https://works.swarthmore.edu/fac-psychology

Part of the Psychology Commons

Let us know how access to these works benefits you

\section{Recommended Citation}

R. Gelman, Frank H. Durgin, and L. Kaufman. (1995). "Distinguishing Between Animates And Inanimates: Not By Motion Alone". Causal Cognition: A Multidisciplinary Debate. 151-184.

https://works.swarthmore.edu/fac-psychology/536

This work is brought to you for free by Swarthmore College Libraries' Works. It has been accepted for inclusion in Psychology Faculty Works by an authorized administrator of Works. For more information, please contact myworks@swarthmore.edu. 


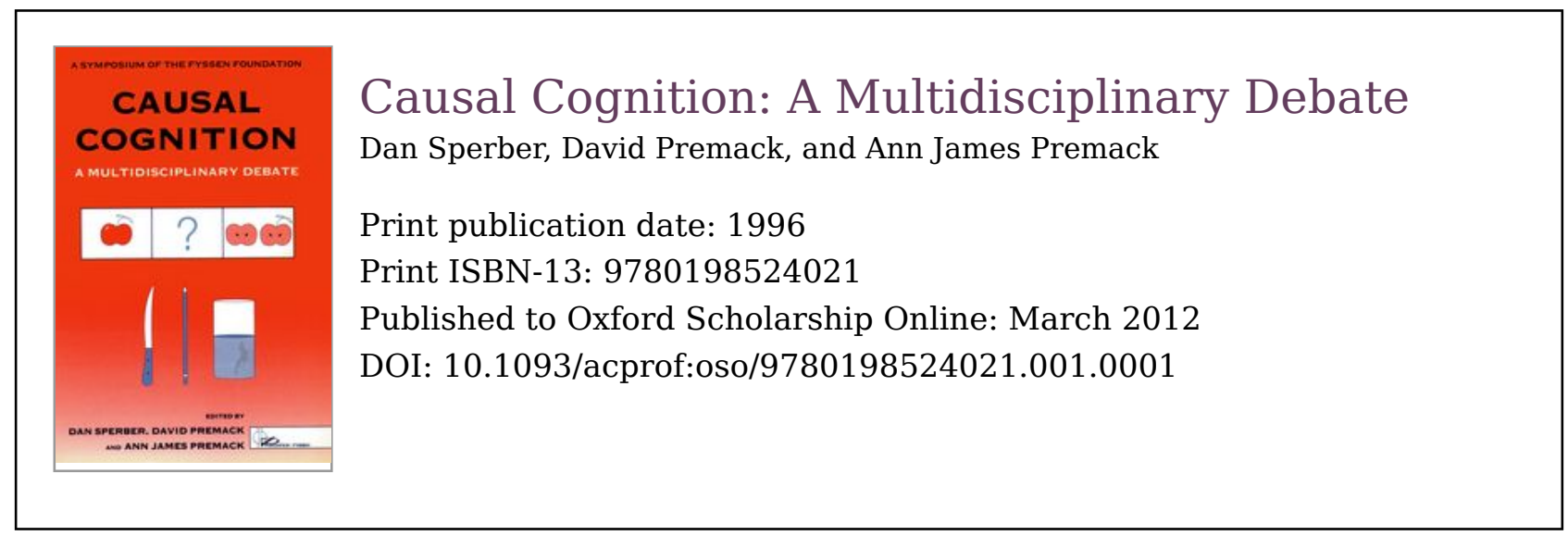

\title{
Distinguishing between animates and inanimates: not by motion alone
}

\author{
Rochel Gelman \\ Frank Durgin \\ Lisa Kaufman
}

DOI:10.1093/acprof:oso/9780198524021.003.0006

\begin{abstract}
Keywords
This chapter presents an account of the origins and development of one's ability to classify moveable entities as either animate or inanimate. The account builds on the known abilities of young infants to find three dimensional objects and to reason about some of their fundamental physical characteristics, for example, that they occupy space, move as a whole, or cannot pass through each other. This chapter also shows that motion paths are ambiguous for adults, not just infants. A moving object is perceived as inanimate when its motion path is consistent with Newtonian laws of motion. If the motion path violates Newtonian principles, then animacy is perceived.
\end{abstract}

Keywords: animate, inanimate, infants, objects, motion, laws of motion, Newtonian principles

\section{Introduction}

In this chapter we present an account of the origins and development of our ability to classify moveable entities as either animate or inanimate. The account builds on the known abilities of young infants to find three dimensional objects (Kellman and Spelke 1983) and to reason about some of their fundamental physical characteristics, for example that they occupy space, move as a whole, cannot pass through each other, etc. (see Chapters 3, 4, and 5). We argue that infants' abilities to find and reason about objects are complemented by skeletal causal principles. First-causal principles lead infants to attend to and interpret 
both an object's composition and its motion path in ways that are relevant to the distinction between animate and inanimate objects. They do so because they support interpretations of motion and transformation with respect to the requisite energy sources and the material types involved in the object's composition and motion path.

Our account of young infants' differential treatment of animate and inanimate objects is fundamentally conceptual and therefore diverges from those that limit infants' abilities to the perceptual level. The latter class of accounts of animacy are based on the assumption that the perceptions of particular kinds of movements can, alone, yield veridical animate or inanimate attributions (Bassili 1976; Mandler 1992; Stewart 1984; Chapters 7 and 20 of this volume). Our account, which focuses attention on abstract causal principles, is not unique. It is consistent with the argument of Heider and Simmel (1944) that the motion paths of objects and their interactions are interpreted in terms of schemata (Goffman 1974; Hochberg 1978). Related views have been developed by Leslie, Talmy, Spelke and her colleagues, other authors in this volume who attribute infants' (and beginning language learners') interpretations of objects and/or semantic relations to force-dynamic conceptual primitives or implicit theories about physics.

(p.151) We favour the idea that an underlying conceptual scheme serves infants' selection and interpretation of inputs for several reasons. Data about motion paths are often ambiguous. In addition, motion path information is neither necessary nor sufficient for the correct identification and interpretation of novel instances. Therefore perceptions about trajectories cannot be sufficient data for the veridical identification of objects and events as animate or inanimate. Further, since correct identifications and attributions occur even when a novel object is static (Massey and Gelman 1988), information about the path is not even necessary. Finally, it is not clear how a perception-only account of the sources of infants' abilities can explain the evidence that infants are capable of causal interpretations of motion paths, whereas these findings are readily explained by a theory that grants infants skeletal causal principles.

\section{On first causal principles}

Gelman (1990) proposed that attending to and learning about relevant characteristics of animate and inanimate objects benefits from certain processing mechanisms. These are informed by causal principles that correspond to deep and fundamental distinctions about an object's composition and its sources of motion or change. Animate objects can cause themselves to move or change; inanimate objects cannot. These differences go hand in hand with the fact that animate objects are composed of biological matter and honour biological principles, whereas inanimate objects are composed of non-biological material and honour principles of inanimate causation. The cause of animate motion and change comes from the internally controlled and channelled release 
of stored chemical energy that is characteristic of biological entities. The cause of inanimate motion is an external force, and there is always a transfer of energy from one object to another, or a conversion of potential to kinetic energy.

Animate motions have a quality of function (purpose). This is a direct consequence of their governance by control mechanisms that makes it possible for animates to respond (adjust) to environments - be they social or non-social - and adapt to unforeseeable changes in circumstances (Gallistel 1980).

The foregoing considerations underlie Gelman's (1990) proposal that learning about animates and inanimates is guided by first principles regarding the energy sources that enable the motion and change of each class of objects. The innards principle draws attention to and interprets perceptual information about those natural objects that move on their own; the external agent principle supports the processing and interpretation of data about objects that move as a function of external energy sources. In general the idea is 'attend to information about sources of energy and their consequences'. In this context, two points deserve comment.

(p.152) First, we do not endow infants with a modern notion of energy, or even any particular theory of energy. Processing mechanisms that are constrained by the innards principle process objects and related events as if certain natural objects have something inside that enables their own motion and change. The principle is neutral with respect to the nature of what a child might think about the 'inside' of self-propellable objects. Since the ideas about 'insides' vary with the belief system of a culture (at a given place and time in history), they must be learned as a function of experience in one's culture. Similarly, processing mechanisms that embody the external agent principle process events as if an external agent contributes to the energy needed for non-biological objects to accelerate and move/change in certain ways. However, the principles do not carry with them a commitment to a particular account of energy or theory of physical causality. Therefore both causal principles are neutral with respect to the ultimate explanation systems developed about animate and inanimate objects. As skeletal principles, they serve to render salient information that pertains to the motion paths and the conditions that support them for animate and inanimate objects. The external interaction principle organizes conceptualperceptual processing of information that is relevant to inanimate objects and their motions. The innards principle organizes the conceptual-perceptual processing of, and learning about, the characteristics of real world animate objects.

Potential information about a source of energy includes information about the entity itself, particularly whether it is composed of living 'stuff and possesses characteristics that are biological. Information about these is yoked to the difference between the innards and external agent causal principles. Causal principles are not applied in the abstract; they are related to the class of entities 
that interact with the principles, just as entities in other domains are related to the principles that define the domain. Given that the material composition of an object is closely related to the energy conditions that support its path of movement through time and space, causal principles help render salient those features of objects that are related to their motions. This can be expanded as follows. Since biomechanical motions are performed only by biological entities, learners will attend to and learn about characteristics of the objects that cooccur with and are relevant to observed motion paths. Similarly, learners will attend to and process those details of inanimate objects that bear on the nature of its motion path. Information about an object's surface characteristics-its smoothness, shape, and size-as well as its source of movement, the barriers that it encounters, its direction with respect to the ground, and so on are all salient. Causal principles render them salient if the observer uses motion cues to classify novel objects and events as animate or inanimate.

(p.153) Evidence that motion paths are causally interpreted by infants Baillargeon and her colleagues have provided evidence that infants interpret motion paths in ways that are consistent with the external agent causal principle. One example is the demonstration by Baillargeon et al (1985) of object permanence in 6- to 8-month-old infants. In this experiment infants saw the same motion path at two different times. During the habituation phase, they saw a screen rotate towards and away from them through a $180^{\circ}$ arc. Nothing was behind the screen. When their interest in the moving screen declined, i.e. when the infants habituated, the stage was set for creating the viewing conditions of the same $180^{\circ}$ rotating screen for a second time. To do this, the experimenter showed infants an object placed to the left side of the screen. While infants watched, the experimenter moved the object behind the screen and the posthabituation phase of the experiment was started. Once again the screen rotated toward and away from the infant. On alternating trials it either traversed a novel $120^{\circ}$ arc in its rotation or the familiar $180^{\circ}$ arc. Given the physics of the situation, the screen should have stopped at about the $120^{\circ}$ position of its rotation, but when it continued through an $180^{\circ}$ arc (by the use of trick mirrors and invisible doors), it contributed to the adult perception of an impossible event, an unseen block being repeatedly crushed and uncrushed as the screen circumscribed the arc. The event is identified as impossible by adults because they are aware that, except in the world of spirits and ghosts, one solid object cannot move through another. If infants are restricted to the perceptual analyses of motion paths, they should see no difference between a $180^{\circ}$ arc rotation shown in both the habituation and post-habituation phases. They should continue to be uninterested in the event, and prefer to look at the $120^{\circ}$ event, which generates a novel perception. However, if infants interpret the motion paths in terms of causally relevant variables, they should treat the second showing of the $180^{\circ}$ event as different from the first. In fact, they attended more 
to the $180^{\circ}$ event, leading to the conclusion that they interpreted the perceptual information about the motion path in ways that we know are causally relevant.

A subsequent series of experiments by Baillargeon (1987) demonstrated that 7month-old infants relate the path of the rotating screen to causally relevant characteristics, for example the height and compressibility of the object to be hidden in the second part of the experimental paradigm discussed above. Infants expected the rotating screen to stop sooner for the taller of two similarly shaped objects that they saw hidden behind the screen. Similarly, given a few seconds to manipulate a hard and a soft compressible object, they expected the screen to stop sooner when it hid the hard object as opposed to the compressible object. The work reported by Baillargeon and her colleagues and by Spelke and her colleagues in this (p.154) volume (Chapters 34 ) provide evidence that even 3and 4-month-old infants attend to and interpret variables that are relevant to the causal conditions underlying the generation of an object's motion path in time and space.

The Baillargeon studies offer some support for our key assumption that motion paths are causally interpreted. As noted by Leslie and Keeble (1987), infants shown film of a launching event behaved as if they took into account the different mechanical roles of two moving 'objects', the pusher (the transmitter of 'energy') and the pushed (the recipient of 'energy'). Again, the relevant data come from the conditions that lead infants to dishabituate following a habituation phase. In this case, two separate groups of infants watched different pairs of events during habituation and post-habituation trials. The first group watched a pair that consisted of a film of Michotte's launching event (Michotte 1963) and its reversal (we denote these events 1A and 1B). The reversal was obtained by running the film of event $1 \mathrm{~A}$ backwards. The second group watched a pair of events, denoted $2 \mathrm{~A}$ and $2 \mathrm{~B}$, that differed from the first pair in the following way. In event $2 \mathrm{~A}$, there was a half second delay between the pusher's reaching the pushed object and both moving on together. Since the delay removes the causal impression of launching, there is no reason to assign causal roles to the perceived objects. Again, the second event for the subjects in the second group was made by running $2 \mathrm{~A}$ backwards.

Leslie and Keeble (1987) habituated infants to an A event and then showed them the B event, to make up what we shall call an AB pair of events. If infants could not interpret the perception of launching with respect to causal roles, there should have been no difference between the ways that infants in the two conditions reacted to their respective reversals. If they could consider the mechanical roles of the objects, then it should matter to them that event $1 \mathrm{~B}$ illustrated an event that was both conceptually and perceptually reversed but event 2B was just perceptually reversed. In fact, infants in group 1 looked longer at their reversed event than did those in group 2 , a result that provides further 
support for our claim that motion paths are interpreted and that causal principles direct attention to information about energy sources.

The foregoing reviews some of the evidence for our central claim that different skeletal causal principles organize attention to and learning about the animateinanimate distinction. The work of Spelke and her colleagues presented in this volume (Chapter 3), which controlled for the fact that animates and inanimates tend to differ in size and surface characteristics, demonstrates that 7-month-old infants know that, whereas two inanimate objects have to contact each other if a causal event is to occur, the same is not true for two people. The authors contrasted infants' reactions to two pairs of videotaped displays. The inanimate pairs of stimuli were two (p.155) objects of heights 5 and 6 feet which had distinctive shapes and contrasting bright colours and patterns. The animate pairs consisted of two people. In the inanimate test condition, infants watched two events: (i) the objects moved towards each other, touched each other, and changed direction; (ii) the objects moved towards each other, stopped briefly before reaching the point of contact, and changed direction. In the people condition the structure of the two events was identical with that of the inanimate events. For example, the parallel event for the person contact condition showed a person holding her arms up and close to her body as she brushed alongside another person. During test conditions, infants looked reliably longer at the nocontact inanimate event; they showed no such preference in the animate event trials. This is what one should observe if the innards and external agent causal principles aid infants in interpreting the motion paths of animate and inanimate objects. Converging evidence that infants interpret a given motion path differently, depending on whether or not the object is animate, can be found in Golinkoff and Harding (1980) and Poulin-Dubois and Schultz (1988).

Evidence that infants interpret the same motion path differently depending on whether the path is generated by an animate or an inanimate object, is particularly troublesome for theorists who favour non-conceptual accounts of initial abilities to respond differently to animate and inanimate objects. For such accounts to work, the spatiotemporal information about the motion paths should not be ambiguous, i.e. it should not be consistent with more than one interpretation. Otherwise, the perceptual inputs cannot lead to the correct classification of an object or an event as animate or inanimate. In what follows we discuss this problem in more detail and show that it is not unique to infants.

\section{Conceptual Coherence: Not by Motion Alone}

Our arguments for a conceptually based account of the early ability to distinguish between animate and inanimate objects parallel those made against the classical definition of concepts (Armstrong et al 1983). The characteristics of redness and roundness are neither necessary nor sufficient identification criteria for a tomato. Both are characteristic of apples, as well as many other objects, and tomatoes can be yellow and oval. To deal with the problems posed by these 
examples, theorists have proposed that concepts are organized around a core implicit theory or set of conceptual principles that function to outline the kinds of information that are relevant to these cores (Carey 1985; Murphy and Medin 1985). These problems do not diminish in an account of concepts of animacy and inanimacy; turning (p.156) to motion patterns as the critical sources of input does not alleviate the problem of indeterminancy. Perceiving that something accelerates in the absence of a source for the acceleration does not guarantee identification of the unknown object as animate, any more than does the perceiving of redness and roundness guarantee correct identification of an object as a tomato.

When perceptual information is ambiguous, one way to disambiguate it is to interpret it within a conceptual framework. The abstract causal principles serve this function for both the infants in the above experiments and the adults in the experiments presented in the next section. They lead one to interpret motion paths in terms of the causal conditions of the motion. Implicit causal principles underlie the information processing that leads to the attribution of causal source(s). They focus processing on those aspects of the situation that are indicative of a mechanical or biomechanical interpretation of the object and its motions and/or the changes it undergoes in form, colour, etc. These include clues about material composition, speed, change in direction, surface characteristics of both the object and its environment, etc.

Animates cannot be distinguished from inanimates simply on the basis of motion because the cues of motion, like the static cues of colour and shape, are ambiguous. A wide range of trajectories can be interpreted as the motions of either an animate or an inanimate object. Our general ability to keep implicit track of the predictive validity of a cue, or combination of cues, leads to knowledge about the kinds of motion paths that tend to go with given object kinds. In a similar way, knowledge about the appearance of objects in each class, the surface cues that tend to distinguish the substances of the two kinds of objects, the environmental factors that are relevant to the motions and reactions of the different objects, and so on is acquired. Such knowledge then functions to help us make educated guesses about the identity of new objects and events. However, a purely cue-driven learning account will not work. Should we discover that our guess is contradicted when we encounter information about the composition and movement conditions of the unknown object, we shall be quick to update our assignment.

Implicit causal principles allow learning like the foregoing to proceed rapidly for the same reason that any learning benefits from available mind structure. Like all mental structures, no matter their origin, causal principles function to combine what is just learned with what is known already. This sets the stage for fast learning about the cues that, on average, distinguish between animate or inanimate items. Armed with such knowledge, even very young children should 
be able to make good guesses about the category assignment of novel items. Evidence that they can is presented later in this chapter. (For further discussion of the parallel function between first principles and acquired structures, see Gelman and Brenneman (1993).)

(p.157) The studies presented in the following sections buttress our position. In the next section we show that motion paths are ambiguous for adults, not just infants. Part of the presentation is based on analyses of some of Stewart's unpublished work $^{*}$ which is being cited in the literature in favour of perceptionfirst accounts (Mandler 1992; Chapter 20 of this volume). This section ends with a report of studies by Durgin and Gelman that reinforce our conclusions about the Stewart data. We then return to developmental issues. A review of preschool children's abilities to assign representations of objects to animate and inanimate categories sets the stage for a discussion of the learning model.

Adult Responses to Motion Paths

Stewart reconsidered and extended

Stewart $(1982,1984)$ proposes that we perceive a moving object as inanimate when its motion path is consistent with Newtonian laws of motion. If the motion path violates Newtonian principles, then we perceive animacy. Attributions such as intentions, desires, hunger, affection, etc. follow. To obtain evidence for her theory, Stewart showed college students a computer-generated ball that moved on the screen in ways that were either consistent or inconsistent with Newtonian mechanics. Examples of these motion trajectories are shown schematically in Fig. 6.1. Some of these are labelled with Stewart's characterization of the animacy cue(s) in a display (e.g. 'Avoidance' in Fig. 6.1(f)). All of them are labelled with Stewart's predictions regarding the expected perception (Animate, Inanimate, Neutral). Figures 6.1(a) and 6.1(b) show one object colliding with another in accord with the laws of elastic collision. Stewart predicted that these would be perceived as inanimate events. However, she predicted that perceptions of displays like Figs 6.1(c) and 6.1(d) would be neither animate nor inanimate but neutral. She argued that an object moving at a constant velocity along a straight motion path offers no information about what initiated the motion and therefore little or no information for animacy or inanimacy.

Stewart's work (Stewart 1982, 1984) is beginning to attract the attention of authors who prefer models of animacy that are not initially conceptual (Mandler 1992; Chapter 20 of this volume). There are results in the work that are consistent with Stewart's account. However, there are some outcomes that are not as predicted; for example individuals do not perceive (p.158) (p.159) an object that moves along the motion path shown in Fig. 6.1(f) as animate. Further, there are possible problems with the data analyses. Stewart assigned categorical judgements to an interval score in some of her statistical analyses. For example in one study subjects responded by choosing between the attributions of 'alive creature', 'non-alive object', 'can't tell' These in turn were assigned degrees of 
inanimacy scores of 0,1 , and 2 for use in parametric analyses. However, 'nonalive' is a predicate that has multiple meanings, including 'dead' which is a predicate that can be used sensibly with animate noun phrases (Carey 1985). Second, it is unlikely that 'can't tell' lies half-way between the animate and inanimate anchors on a psychological scale of animacy. Instead, it is possible that a mean score of 1 masks the fact that some subjects used the animate category and others used the inanimate category. Additionally, the requirement that subjects classify a display into one of the conceptual categories could have encouraged interpretations that fit within this classification scheme; if so, it is hard to exclude the possibility that subjects interpreted what they saw to achieve their answers.

Stewart was well aware of these concerns. Indeed, the rating criterion problem led her to perform a series of unpublished studies on the effects of instructions. In one, she used an open-ended response mode and asked college students $(N=21)$ to report 'what came to mind', what the display 'reminded them of. In subsequent studies, Stewart encouraged her subjects to 'see' all the represented objects and their motion paths in a certain way. In one of these studies, 10 additional students were told that everything represented the motion of an animate object, generated by a person or an animal; in another study with yet another sample of 10 , subjects were told that

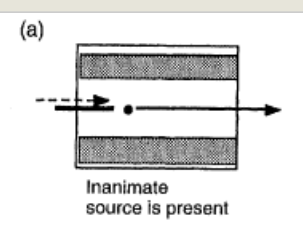

(b)
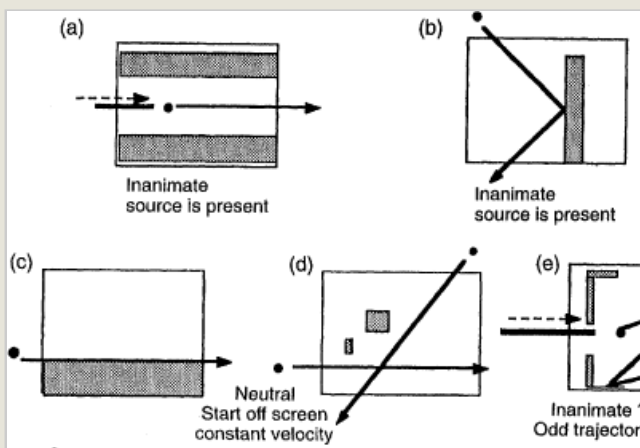
source is present
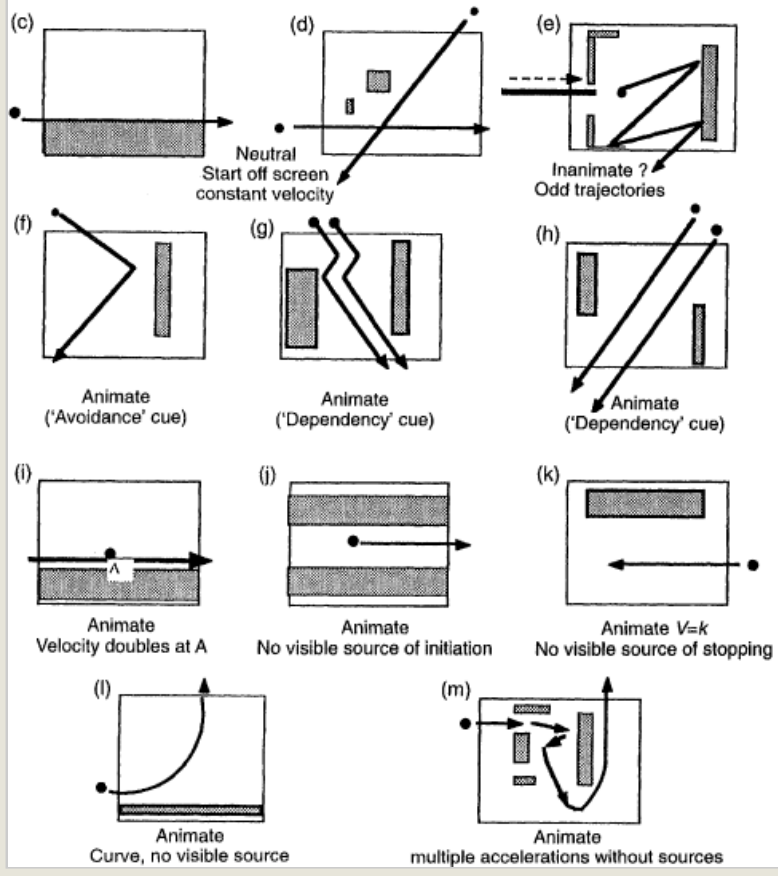

Fig. 6.1 . everything they would see represented the motion path of an inanimate object such as a ball. Fortunately, the same displays were used across all three of these instruction conditions, which we denote here the unconstrained, animate constrained, and inanimate constrained groups. Stewart's generosity with her data makes it possible for us to present analyses of these, the outcome of which is a body of evidence that illustrates the ambiguity of objects' trajectories. 


\section{The new Stewart studies}

The subjects in the unconstrained, animate constrained, and inanimate constrained groups saw the displays shown in Fig. 6.1. The stimuli were created and shown on a Commodore computer. The program first painted between 0-4 orange background strips in haphazardly selected places around the outer portions of the screen (these appear as black bars and squares in Fig. 6.1). When this was complete a black roundish ball (one sprite in size) followed the trajectory programmed for that trial. Sprites moved across the screen at a constant velocity of about 1 inch per second. Accelerations either (p.160) doubled or halved the speed of the sprite. The total duration of a display varied from 6 to 25 seconds. Answers were recorded for later transcription. We decided to code only the first response recorded, on the grounds that these were the least likely to reflect interpretations (our hypothesis) as opposed to cue-driven perceptions (the bottom-up hypothesis).

\section{Coding}

Each respondent's first reply for each display was scored in two ways, first with respect to the kind of object seen and then with respect to the kind of event reported. For the object-kind, we coded whether the noun or pronoun used to describe the ball (actually, the computer sprite) referred to an animate, inanimate, or mechanical object (e.g. tourist, ball, and jet plane respectively). A fourth category, other, was used when an answer was ambiguous, incomplete, or did not fit into one of the above three categories. Examples of other answers included graph, computer game, and skating. Skating was ambiguous because it was not clear whether the observer was talking about a person who was skating or just the skates. When an answer referred to a well-known inanimate event, as when many subjects described the Michotte-like collision in Fig. 6.1(b) as 'pool' or 'a pool game', we assumed that the object was not stated owing to conversation constraints against stating the obvious. In this case, we assumed that had the subject referred to an object, it would be the standard one that fitted the known game or script. In the example under discussion, this means that our object-kind code scored the response as inanimate as opposed to other.

Codes of event-kinds were based only on the kind of motion path ascribed to the display. In some cases, this is all that a respondent talked about, for example a tennis ball hitting a wall. In other cases, the event was embedded in a more complex script, for example where the curved trajectory display (Fig. 6.1(e)) led an observer to say that someone was carrying a balloon that 'was released'. In the latter case, both the object-kind and the event-kind were scored as inanimate. The fact that the perceived motion path was embedded in a causal account is clearly of interest. However, since the latter referred to entities that were not part of the display, to code the perceived motion path itself as animate would be to confound issues of perception and interpretation of the display. We shall return to the issues raised by this example. 
When we scored event-kinds, it was necessary to expand the criteria for the other category to include complex descriptions of computer games ('that's an old Pong computer game'), mathematical and scientific talk about the displays ('a drawing of a parabolic curve' or 'It looks really sticky... when it hits every wall it's not using the laws of physics'), comments on the displays, metaphorical answers ('a stable relationship'), and literal descriptions ('It moved along a diagonal, then it moved in another direction').

(p.161) Use of the event-kind code for a description was usually straightforward, as for That person is going fast', which was classified as animate. However, there were problem cases, particularly in data sets from the two constrained instruction conditions. For example, one subject in the inanimate constrained group said that the display in Fig. 6.1(m) was 'a vacuum cleaner doing a lousy job', and subjects in the animate constrained group told us about animate objects being bounced or pushed through walls after seeing collision displays. In these cases, although the case of the noun phrase (NP) and verb phrase (VP) do not agree, one senses that the VP encodes the intended answer. It is as if the respondents have found a way to meet the demand characteristics of the task and at the same time tell us what they 'really' saw.

Three lines of evidence supported our decision to focus on the VP when classifying reported events as animate, inanimate, mechanical, or other. First, verbs are often very selective about the meaning of the nouns with which they pair (Pinker 1989; Gleitman 1990). For example, if we hear That dax is frightened', The zifs are walking', or The tral is investigating', we are inclined to assume that the NP is animate. These facts justified our decision to accept psychological verb phrases (ones that referred to knowledge states, volition, desire, motivation, motivational states, emotions, perceptions, and goal-oriented plans of action) as evidence that subjects saw an animate event, despite their use of an inanimate NP. Therefore, although the it in 'it knew where it wanted to go' and 'It gets frightened' was coded inanimate in the object-kind analyses, the events in which the object participated were scored as animate.

Use of sociolinguistic devices can mitigate or exaggerate the animacy of an agent. This can be accomplished by using the verb in the passive tense as did the animate constraint subject who said The child was thrown' after watching a collision display. In addition, English speakers can use indefinite pronouns, or 'you' and 'they', in an indefinite function to depersonalize or render an agent neutral or nondescript, for example They were pushed'. Results of this kind (Weiner and Labov 1983) supported our decision to pay attention to the details of verb use when coding for event-kind. See also Beedham (1987), Lamb (1991), and Stanley (1975). 
Finally, we obtained new data to validate our reliance on the VP in order to classify descriptions in which the class (animate, inanimate, etc.) of NP and VP disagreed. We presented a group of introductory honours psychology students at the University of Pennsylvania with a variant of the fill-in-the-blank task. In our version, passages with a blank occurred in the NP. Some of these were created by deleting the item(s) that were in conflict with the structure and semantics of the VP. For example That is a vacuum cleaner doing a lousy job' became That is a ----- doing a lousy job', 'The ball couldn't decide' became The ------ couldn't decide' and The animal (p.162) is being thrown' became The ----- is being thrown'. Others were created by deleting material in the NP that was consistent with the VP, for example 'a leaf being blown around' was rendered 'A was blown around'. These control sentences were included to verify our judgement that the NP and VP classes agreed semantically and structurally.

As expected, all 21 students filled in the vacuum item with animate nouns, including worker, janitor, student, cook, businessman, carpenter, and painter. Similarly the original The ball couldn't decide' generated nothing but animate answers, including man, boy, debater, mother, Danish Prince, judge, and jury. The original The person was bounced off something' was paired with nouns that were all inanimate except one, a bird. Answers included ball, rock, golf ball, superball, and spring. Finally, the example control sentence elicited nouns that were referents for light objects like leaves, feathers, etc. These data were used to check our coding assignment on the basis of the linguistic cues detailed above or when such cues were not present because subjects had compromised the syntactic and semantic rules of agreement.

Coding of both the object-kind and event-kind was reliable. A sample of 54 per cent of the data was scored twice, once by one author in consultation with an independent rater and once by another author. Agreement between raters was obtained for 93 per cent of scored object-kinds and 92 per cent of the eventkinds. The raters continued to score the remaining protocols on their own until all were coded. Unless noted to the contrary, the results presented below are based on event-kind data. There are two reasons for this decision. Focus on the event-kind analyses is probably more consistent with Stewart's scoring procedure; it appears that her results were based on codes that included details regarding event descriptions. Second, as already indicated, the object-kind answers were more likely to reflect demand characteristics. For example, when animate constraint subjects saw the odd collision path (Fig. 6.1(e)), all but 20 per cent complied with the instructions to interpret the display as animate by giving an animate NP but 60 per cent generated inanimate event descriptions.

\section{Baseline considerations}

The overall pattern of results from the unconstrained group are shown in Fig. 6.2. They are consistent with Stewart's earlier reports which means that we can treat them as a baseline against which to compare results from the groups that 
were constrained by instructions to attempt to give animate or inanimate interpretations. In this section we ask whether the detailed pattern of results matches the predictions made by the Stewart model. To anticipate, there are enough departures for us to conclude that the results are better accounted for by the kind of account that we favour.

Figure 6.2 shows the percentage of subjects who offered a given kind of (p.163) event description for each display. The displays are arranged from bottom to top in order of their tendency to elicit animate descriptions. Clear collision events were seen as inanimate by most of the subjects and never elicited animate attributions. The display that contained multiple stops and starts in the absence of any source for these was the one most likely to elicit animate attributions. The displays with a mid-point acceleration or paired and co-ordinated motion paths were reasonably good inputs for animate attributions.

Although the foregoing details of the results are all consistent with Stewart's account of animacy, others are not. Contrary to Stewart's prediction, the display that traced a collision 'avoidance' path (Fig. 6.1(f)), i.e. did not hit a barrier, was not particularly seen as animate. Many of the motion paths that violated Newtonian mechanics were

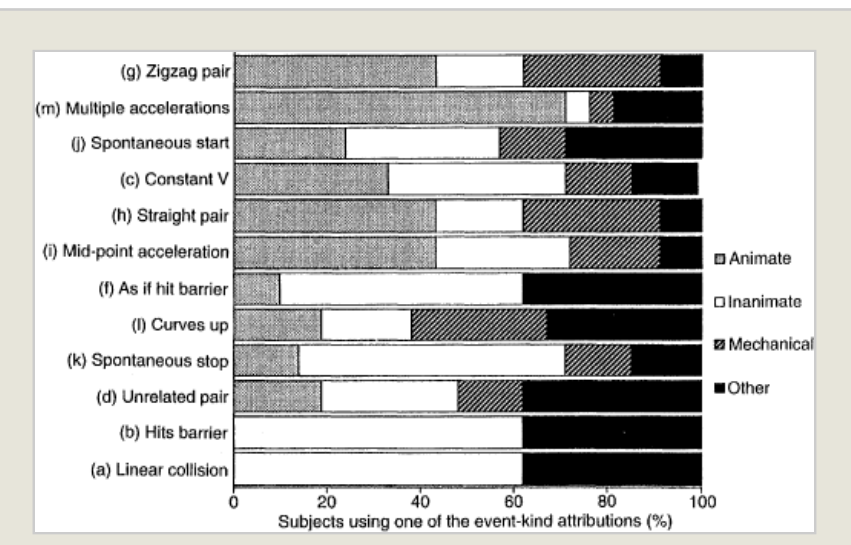

Fig. 6.2 . ambiguous: some subjects saw them as inanimate and some saw them as animate. This is not what is expected if the perception of animacy is like 'a perceptual illusion' (Stewart 1982) and therefore not subject to conceptual interpretation (cf. Chapter 5). Two displays should have been preferentially judged as animate-the one that showed an object traversing an upwardly curved motion path (Fig. 6.1(1)) and the one that showed the object starting to move on the screen without a causal source (Fig. 6.1(j)). However, they (p.164) elicited animacy judgements in only 25 per cent and 20 per cent of the subjects respectively.

Some might argue that there is a way of viewing the problematic data points we cite that will be more consistent with Stewart's model than we allow. Perhaps 'mechanical' attributions are used for weaker animate percepts. The idea would be that perceived mechanical and animate events are interchangeable; both solve the perceptual problem of accounting for acceleration in the absence of an external source. However, given the subjects' use of mechanical scripts, it would be a mistake to collapse these. Consider the data for displays where the object started to move on the screen (Fig. 6.1(j)) or accelerated at the mid-point (Fig. 
6.1(i)). Although some observers gave mechanical attributions to a display that should be animate, others used inanimate attributions. As can be seen in Fig. 6.2, this tendency is particularly strong when the display object started on its own (Fig. 6.1(j)) or stopped without slowing or contacting another object (Fig. 6.1(k)). More generally, there is too much variability across displays in the use of mechanical event accounts to hold that these are interchangeable with animate attributions. These considerations, together with others presented later in this chapter, encourage us to keep the mechanical and animate categories separate. The evidence fits better with the conclusion that machines are in a hybrid category of their own.

The fact that so many of the Stewart displays are best described as ambiguous is consistent with our account. The information from a limited segment of the motion path of a novel object is often insufficient to render an attribution. The perceptual information is interpreted with reference to the possible causal conditions consistent with what is observed. When a motion path is not unique to a class of objects, we should expect more than one outcome of the interpretative processes. For example, as our subjects showed, the perception of a particular curving path lends itself to a wide range of causal interpretations including '...a cyclist going around a corner','... a balloon and the wind was blowing and it went like this, this, like a helium balloon. It got caught up in the air' , '...a horse climbing a mountain', and '...some kind of magnetic ball that encountered a field that pushed it away'.

The results from the display where there was an abrupt collision-like change in direction even though the ball never made contact with the barrier (Fig. 6.1(f)) are particularly interesting. Contrary to Stewart's prediction, subjects treated this as inanimate. In our view this is what they should have done because the trajectory does not have high validity for an 'avoidance' event. In avoidance paths, it is seldom the case that the angle of incidence is equal to the angle of reflection. This is characteristic of elastic collision events. However, the anomalous causal information means that observers should give reasonably novel interpretations of the event or even suggest (p.165) that they misperceived it. In fact, many individuals either noted the fact that the ball failed to hit the wall, appealed to invisible barriers or clear walls, or suggested that electromagnetic forces were at work. Some asked why the ball did not hit the barrier and one person claimed that his eyes were playing tricks on him.

Our position differs from Stewart's in another rather subtle way. We have no reason to treat trajectories that offer little information about the animacy or inanimacy of an object as neutral, i.e. they will lead to descriptions that are neither animate or inanimate. Instead, such trajectories should be particularly ambiguous, i.e. they should encourage rich interpretations that supply considerable detail to relate the perceptual input to a causal account. This is what happened with the Stewart neutral displays (Fig. 6.1(c)) and 6.1(d)). 
Display 1(c) led to accounts like 'some kind of train going along a track', 'A swimmer swimming in a pool', '...something going over ice...like scooting over ice', 'Something rolling along in a gutter', 'A rabbit running along, like when you see the horse races, and the rabbits on the fence...', and 'A father sending a ball across the floor'.

These responses embed within them an account of how the seen object could have moved at a constant velocity, either because of a tracking device, characteristics of the surface, or a particular kind of actor in a particular kind of setting. This is the kind of evidence that we need to conclude that the data are not neutral-eliciting no impression, one way or another-but are instead fodder for the causal principles, encouraging interpretation of what is perceived in terms of the possible agents and conditions that generated the trajectory. Put another way, this is further evidence for our belief that attributions of animacy reflect the workings of mutually constraining perceptual and conceptual processes. We can expand this as follows.

Individuals' perceptions of the spatiotemporal characteristics of the motion paths (trajectories) shown schematically in Fig. 6.1 were almost always veridical in the unconstrained group, i.e. object-kind and event-kind attributions were consistent with what was shown regarding the speed, acceleration, and path of the object. However, the attributions were not based solely on these characteristics. What was perceived was interpreted with reference to the conditions that could cause such a trajectory, even if it meant inventing invisible barriers, unseen tracking devices, and meanings for the rectangles and bars that happened to be on the screen. In other words, veridical perceptions were related to ideas about the kinds of environments in which an event could have occurred. In turn, all this was related to causal considerations, i.e. to one's hypotheses about the kinds of things and conditions that could cause the trajectory in question. Thus causal principles constrained what was perceived. Conversely, causal interpretations were constrained by what was perceived. For example, the (p.166) interpretative freedom given the perception of an object rapidly traversing a path wherein the angle of incidence equals the angle of reflection was less than the interpretative freedom allowed by an object moving at a constant velocity. Therefore we conclude that the causal interpretation of the data is deeply related to the input, even though what is perceived does not, on its own, generate a perception of animacy or inanimacy. The results from the groups whose interpretations were constrained by our instructions support these ideas.

\section{Constrained instructions as setting conditions}

If, as Stewart suggested, the perception of animacy is akin to an illusion, then telling someone to 'see' such events in another way should have no more effect than do instructions not to see the Muller-Lyer illusion. However, if perceptions of animacy and inanimacy are both constrained by and able to constrain causal 
reasoning about the data, there are conditions under which instructions should alter respondents' reports. If subjects are asked to 'see' a display as animate, they should be able to do so if this interpretative set is consistent with the constraints given by the perceptual inputs. Otherwise, they should not succeed in meeting our request. More generally, individuals' success with constrained instructions should be related to the extent to which a display includes information that is consistent with the request. Therefore individuals should find it harder to 'see' motion paths that typically index collisions as being associated with animate events. Similarly, observers should have difficulty in finding inanimate solutions for displays with multiple accelerations. In contrast, they should be able to find animate solutions where an object's motion path stops in the middle of the display, even though the baseline bias, as revealed in the unconstrained group, is for an inanimate event. This is because the data include information that is relevant to the abilities of inanimate objects to start and stop themselves. Put differently, the instructions can be thought of as setting subjects to pay attention to those aspects of a display that are relevant to a certain kind of causal account. Subjects should follow instructions better when given displays that contain more such features.

The top and bottom panels of Fig. 6.3 summarize the results from the inanimate instruction and animate instruction groups respectively. As can be seen, observers in both groups responded as expected. They were not very good at finding acceptable animate solutions for collisions (Figs 6.1(a) and 6.1(b)); similarly, they resisted the experimenter's request for an inanimate solution for the display with multiple accelerations (Fig. 6.1(m)) and twinned zigzag trajectories (Fig. 6.1(g)). Otherwise, they were rather adept at 'seeing' displays in ways that met the constraints of the instructions that they received. We end with some samples of what kinds of events (p.167) observers reported under these experimental conditions. More importantly, they actually work; that is, they reflect solutions that involve a combination of a veridical perception and a causally acceptable interpretation. They illustrate our point that abstract causal principles focus attention on information that can be related to a reasonable causal account of an object's movements. 
Examples of some of the answers from the constrained conditions help to illustrate this point. Answers (a) and (b) were obtained in response to the trial during which the sprite moved horizontally across the screen and (p.168) seemingly stopped on its own (Fig. 6.1(k)). As can be seen in Fig. 6.3, this was not a very animate display for unconstrained subjects.

Answers (c) and (d) are examples that satisfied the constraints of the multiple acceleration display (Fig. $6.1(\mathrm{~m}))$ as well as the inanimate instructions.

(a) It looks like my mum shopping and stopping right in front of a store.

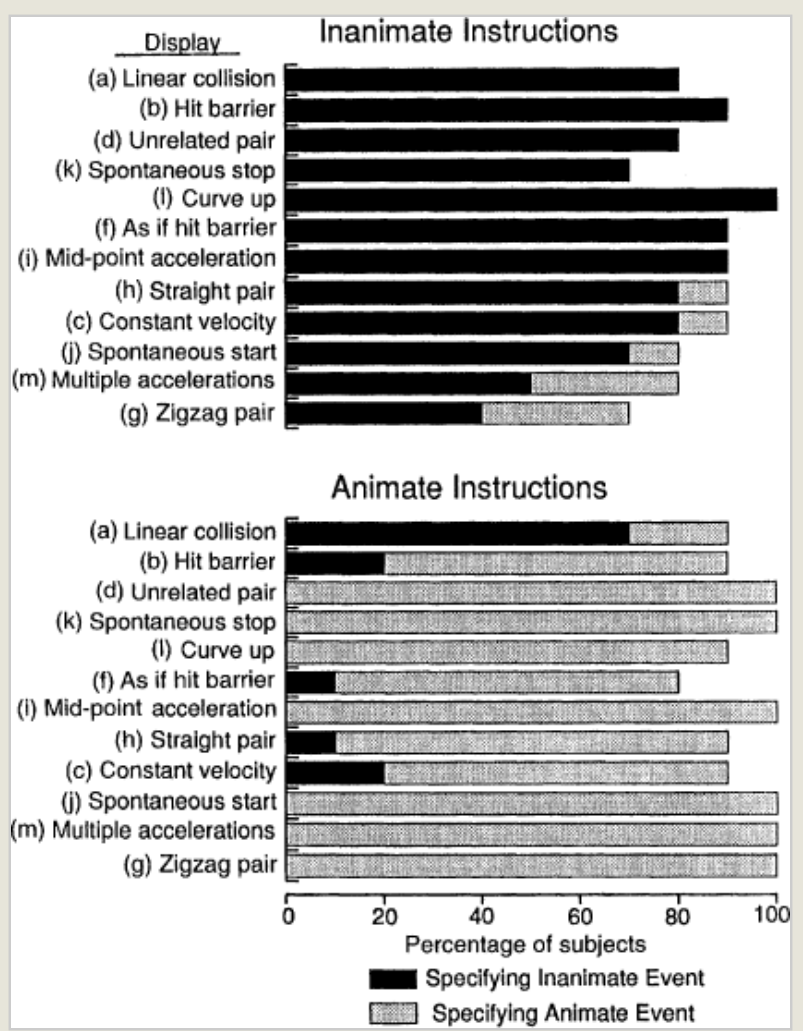

Fig. 6.3 .

(b) That was a figure skater

that fell down.

(c) (long pause). Gosh, I don't know...let's try just a windblown object.

You're in an area where it is swirling and stopping and changing

direction....

(d) Kind of reminded me of some sort of balls in a track that can only go in certain....

All these examples include clear information about the causes of one or more accelerations, despite the fact that no such information was represented in the motion path: someone's mother stops abruptly to look at a shop window; a skater falls unexpectedly; a path which usually has high validity for animacy is rendered inanimate by an unseen wind or tracking device, the kind of causal agents that can cause erratic changes in direction. Similar features characterize the Durgin-Gelman transcripts discussed below, even though they were obtained with somewhat different displays that moved more slowly across the screen.

\section{The Durgin-Gelman studies}

The studies presented here support our conclusion about Stewart's data-that attributions of animacy reflect the operation of mutually constraining perceptual and conceptual processes. Principles of causality direct and modulate attention towards relevant aspects of objects and their motions. They conceptually constrain the interpretation of novel objects such that those objects with cues 
that have high predictive validity for animacy are likely to be classified as animate, while those with characteristic cues for inanimacy are more likely to be given inanimate attributions. Furthermore, the 'cues' suggesting animacy or inanimacy are sometimes more conceptual than perceptual (e.g. goal-directed activity). The film recorded by Heider and Simmel (1944), which is often cited by those favouring a direct perception account of animacy, shows a rich supporting environment that clearly constrains answers, for it is unlikely that talk about people chasing each other in and out of a house would be forthcoming if the shapes were not moving in and out of a square with an opening. Interestingly, much of the supporting role of the static environment is de-emphasized, or even ignored, in bottom-up theoretical accounts (Bassili 1976). The present studies were undertaken to investigate the possible interaction of a moving object and (p.169) its environment under conditions in which interpretation is unconstrained by perceptually available information. Our studies thus bear on our interpretation of Stewart's findings, and on the role of conceptual principles that we believe are used in organizing perceptual evidence.

The Durgin-Gelman studies have much in common with the Stewart studies. Like Stewart, we presented computergenerated displays of one or two small balls (sprites) moving on a video terminal* and, again like Stewart, we collected both descriptions (unconstrained) and rating data. However, we used a combinatorial design to analyse the effects of various features and we adopted a continuous rating scale of

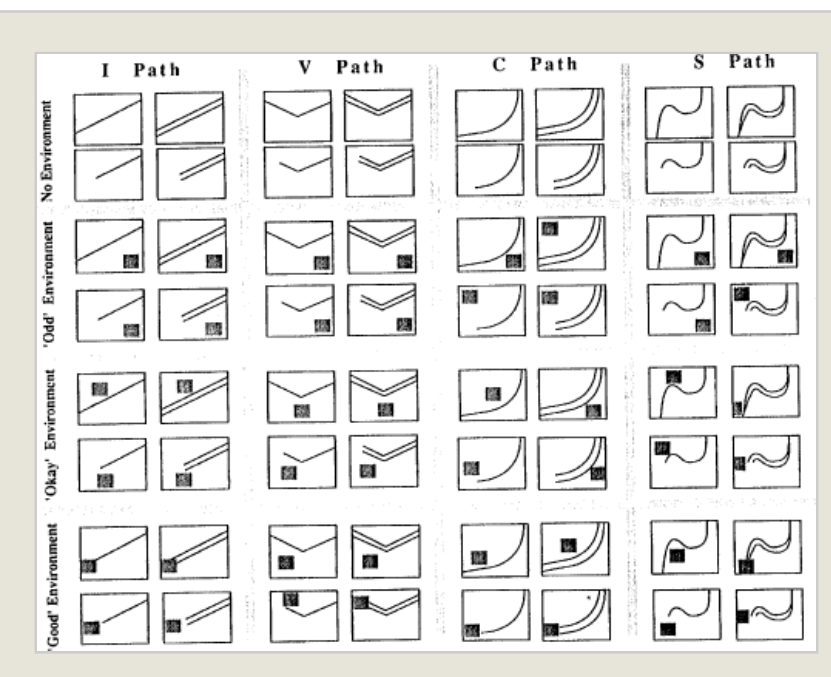

Fig. 6.4 . animacy to supplement our coding of verbal descriptions of the displays. Schematic drawings of the 64 displays are shown in Fig. 6.4. The design for the creation of these displays called for the combination of straight or curved segments with or without inflections to produce four types of trajectories which we label, (p.170) according to their shape, I, V, C, and S. The four types of trajectories were combined with combined with three variables: (a) four levels of environment quality (none, odd, okay, or good) defined by the presence of a single grey rectangle at some location on the screen, (b) stopping on or moving off the screen or not remaining on the screen, and (c) number of sprites (one or two). 


\section{Design details}

Because the displays are rather similar, we decided against a full factorial experiment. Instead, a quasi-latin-square design (Cochran and Cox 1957) was used to present the 64 displays according to a balanced plan, which meant that we presented subjects with a different sample of eight displays in each phase of the experiment. Fifty-six subjects (undergraduates from the Universities of Pennsylvania and Virginia) first gave descriptions of eight displays (phase 1). Forty of these subjects then gave ratings of animacy to a different set of eight displays (phase 2). Thus each of these participants saw, at most, 16 displays, and each of the 64 individual displays was described by seven subjects and rated by five (different) subjects.

The two-phase experiment lasted about 35-40 minutes. The first phase required an open-ended response and therefore paralleled the Stewart unconstrained condition. The second phase required ratings of the degree of 'aliveness' of the object/event shown in each display. The description task was presented first, so that the explicit animacy instructions of the rating scale task did not bias the open-ended responses. By saving the rating task until last, we circumvented the need to familiarize subjects with samples of the displays to anchor the points on the rating scale. Subjects were alone during the study; they were run in by and responded to a computer-generated experiment. All stimulus presentation and data entry was subject controlled. Individuals typed their phase 1 attributions on the computer keyboard, and 'painted' their phase 2 ratings (from 'not at all') to Very alive') on the screen using a mouse. The length of the bar gave us scores that ranged from 0 to 400. The overall mean animacy rating was 158 .

\section{Some expectations}

Our primary expectation was that interactions, in both senses of the word, would be important. Animate objects can respond and adjust to their environments in ways that are unavailable to inanimate objects. Thus, although we expected objects moving on curved trajectories to evoke a higher animacy rating than those moving on straight paths, we also expected that more meaningful environments would add to the impression of animacy (by meaningful environments we mean environments that support interpretations of a moving object(s) interacting with its environment). However, the presence of inflections, or changes in the direction in which the object was heading (as in the S- and Vshaped paths), was expected to (p.171) interact with the local path shape (curvilinear or rectilinear) because the inflected straight line which formed the V-shaped path contained information specifying a collision which was countersuggestive of animate attributions. Moreover, we have learned from Stewart's data that environmental variations have little effect on attributions given to collision-like trajectories. The V-shaped path is so characteristically inanimate that Stewart's observers preferred to invent environments in order to 
explain better a causal script involving collision rather than assimilate it to an animate event involving avoidance.

Given that co-ordinated activity is much more characteristic of animate action principles, we also expected that path twinning would encourage animate attributions and boost animacy ratings. However, once again we were prepared for interactions between this variable and others, for we expected individuals to integrate all information when interpreting their perceptions. If they have perceptual inputs that are particularly

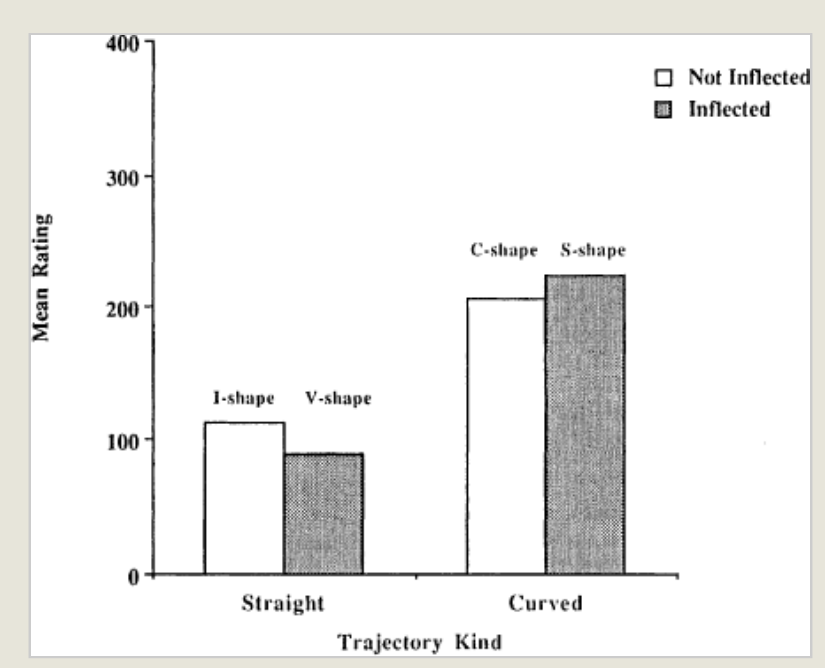

Fig. 6.5 . characteristic of inanimate events, then they might well find an inanimate causal script that is consistent with both this information and the presence of twinning. It should be remembered that Stewart's subjects could assign inanimate scripts to coordinated motion paths. They did this by appealing to causal conditions like tracking devices and transparent connectors.

\section{(p.172)}

\section{Rating data}

The effect of segment shape (curvilinear versus rectilinear) on the animacy ratings, which is shown in Fig. 6.5, was significant $(F(1,39)=170, p$ $0.01)$. The anticipated interaction of the presence of an inflection and segment shape was also found $(F(1,39)=5.6, p$ $0.05)$, reflecting the fact that the presence of an inflection enhanced the rated animacy of the curved path but decreased

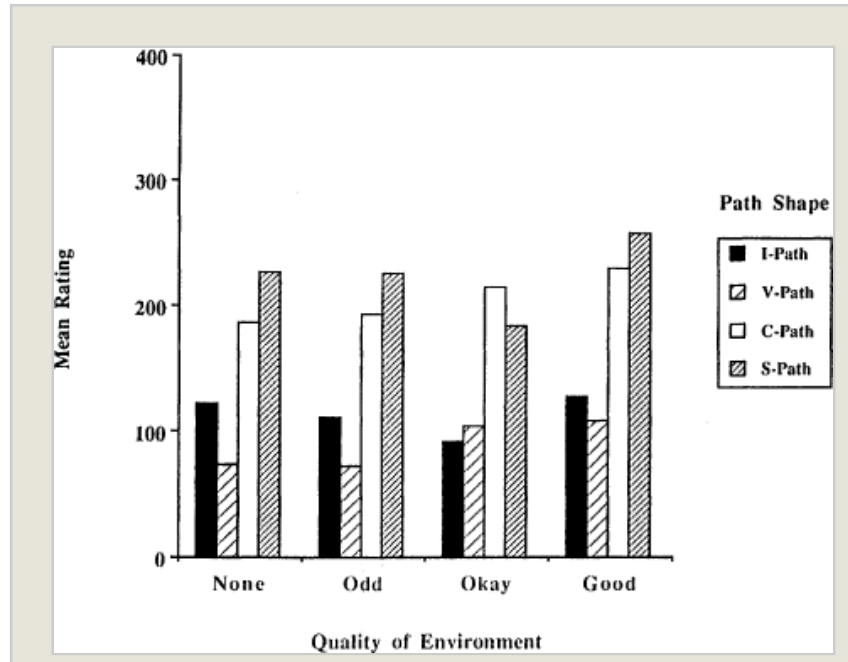

Fig. 6.6 . the rated animacy of the otherwise straight path (Fig. 6.5). More important to the main hypothesis of the study, there was a significant environmental effect (Fig. 6.6), which is reflected in enhanced animacy ratings provided by the 'good' environment $(F(3,37)=3.8$, 
$p$ 0.05). Moreover, there was a significant interaction between path type and a portion of the environment variable $(F(3,37)=5.0, p 0.05)$ which can best be understood by examination of Fig. 6.6. Although the odd environment is the same as an absence of environment for all types of path, the l- and S-shaped paths are extremely sensitive to the relative goodness of the 'okay' and the 'good' environments. Both show a relative decline in animacy when the environment is only 'okay'. There were no main effects of stopping or of having more than one object. However, there were patterns of interaction involving these variables, which we shall discuss below.

\section{(p.173) Free-response data}

The descriptions produced during phase 2 of the experiment were coded according to the scheme developed for the Stewart studies. As shown in Fig. 6.7, the pattern of animate event attributions across the four kinds of trajectories are consistent with the rating results. Observers were most likely to attribute animate events to curved paths and inanimate events to V-shaped paths. Despite these tendencies, all trajectories were ambiguous. Even though S-shaped paths received more animate than other kinds of attributions, the fact remains that some people did interpret these as evidence for an inanimate event, for example 'Moves similar to a feather caught in the wind' or 'A smoke particle as seen from above in a wind tunnel' or 'a falling snowflake Similarly, some observers paired V-shaped paths with animate events, for example bird flying above a house' or 'A person walking up to a building to see if it's open. It wasn't so he walked away' or '...a vulture flying down to the ground to get its prey'.

The fact that even the curved displays were ambiguous is consistent with an important feature of the rating data. Although the displays in question were judged to have reasonable cue-validity for animacy, they did not receive particularly high animacy ratings. This is what one would expect if simple trajectories like ours and Stewart's do not, on their own, guarantee an animate percept. Intermediate level ratings are consistent with our view that information can have reasonable cue validity for animacy without being defining.

Attributions, like ratings, were influenced by the expected interaction between kind of environment and path direction. To show this, we start with distributions of animate and inanimate attributions for the four different environments. As can be seen in Fig. 6.8, subjects favoured animate over inanimate interpretations for okay and good environments. In contrast, inanimate attributions dominated, given either an odd environment or no environment. Animate interpretations for an object that moved in the context of an okay or good environment included 'A bee going into a bee hive' or 'A baseball player running back to the dugout'. These are very different in character from the two animate attributions that were assigned to the same motion path shown without an environment or with an odd environment. In neither case do the actors move on their own: 'Someone riding a sled down a steep slope' or 'It looked like an object, for example a skier, 
headed down a steady incline'. Instead, they use an inanimate object that is suited to the presumed inclined plane in the environment. No such crutch appears in the attributions offered when the same motion path was set in a Good environment. The scripts generated here always involve self-initiated goaloriented actions that interact with a particular place. These qualitative differences are particularly interesting and help (p.174) (p.175) account for the relationship between environment quality and event-kind attributions.

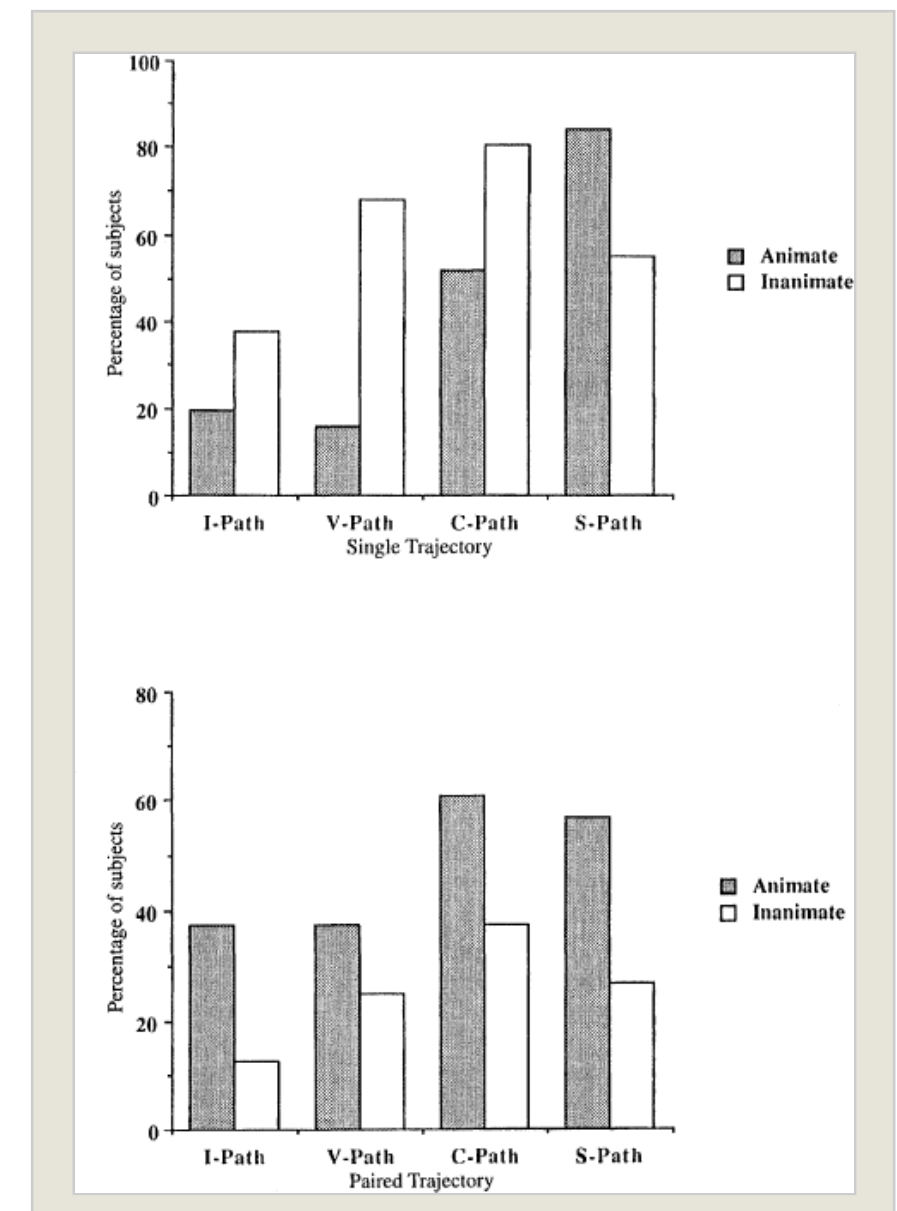

Fig. 6.7 . 
The capacity for self-generated actions is closely related to the ability to respond and adjust to the environment. Hence, when a moving object is in an environment in which it might come close to but avoid, enter, etc. an object, the constellation of information should be readily assimilated to interpretations that are consistent with the innards principle. Therefore, on average, attributions should be more animate. For similar reasons, given a path that has good cue-validity for inanimacy,

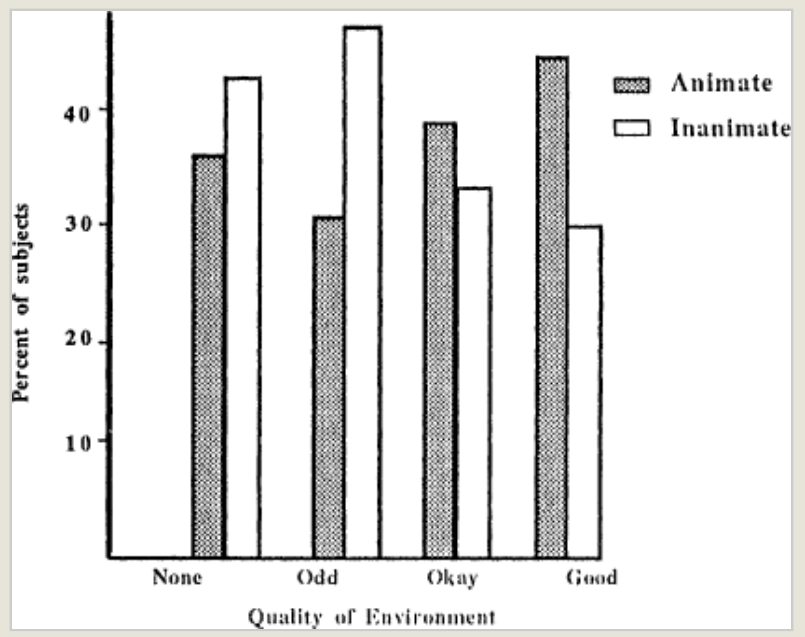

Fig. 6.8 . The effects of environment on event attributions in the Durgin-Gelman Study. the external agent inanimate principle should encourage attention to those aspects of the environment that bear on the calculation of its speed and trajectory, for example the surfaces of an object, the medium on (in) which it moves, its relative position with respect to the ground, its size, and its shape. Interpretation of these inputs can proceed with a rather narrow sampling of the environment around the path. If nothing is proximate, as in the odd and no environment conditions, there is no reason to alter an ongoing interpretation of the perceived information. Therefore it is not surprising that ratings of inanimacy are more likely when there is no environment or the environment is at a distance.

Our account of the quality of environment effect also applies to why twinned motion paths should, on average, encourage animate ratings and (p.176) event-kind attributions. When the environment is social, animates can respond in kind, co-ordinate their acts with others, oppose others, and so on. Ratings reflected an interaction between change of direction and pairing. The qualitative effects of path number on event-kind attributions as a function of path shape are shown in Fig. 6.9. 
Figure 6.9 shows the overall tendency for observers to write about animate as opposed to inanimate events depending on whether the display had one or two paths and whether the path was straight or not and changed direction or not. Since the rate of mechanical attributions was comparable across conditions, only animate and inanimate attributions are represented in the figure. Setting aside consideration of the S-shaped

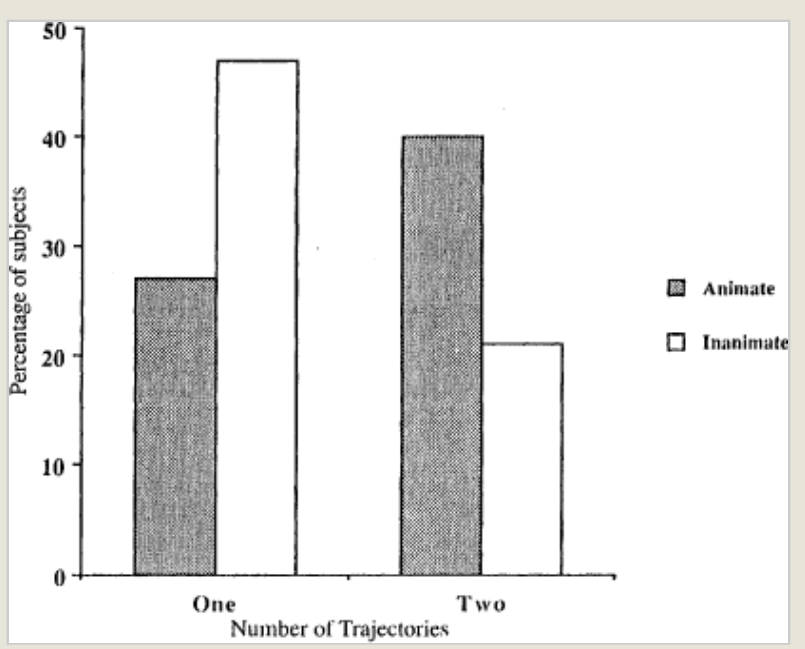

Fig. 6.9 . path findings for the moment, a common generalization can be made about the data shown in Fig. 6.9. The single versus pair variable had the effect of switching observers from a preference for inanimate attributions to a preference for animate attributions. For example, although observers favoured inanimate attributions for a single C-shaped path, they preferred animate attributions for twinned C-shaped paths. However, as with our rating results, the extent of the pair effect is not constant across the different path shapes. The different pattern of answers for the straight and V-shaped paths illustrates this point. Further, although animate attributions were favoured for both single and paired S-shaped paths, it is important to point out that the overall (p.177) level of animate attributions was depressed by the paired displays. Results like these tend to exclude models that assume that cues for animacy are additive (see Chapter 20), particularly because subjects' comments show that the repeated finding of interactions between variables indexes a strong tendency for individuals to relate and interpret their percepts to causal principles. Individuals' attributions are full of comment about the speed, weight, size, and surface characteristics of an object. In turn, these are placed in media (usually invented) and related to environments that can support the observed characteristics of the trajectories.

In summary, the characteristics of the trajectory influence the interpretation, but they do not determine it. The interpretation of the event is generated by the interaction between the principles that guide a causal analysis of the event and the characteristics of the observed trajectory.

Preschool Children Can Assign Animate and Inanimate Predicates to Still Photographs of Novel Stimuli

We have been developing the position that, on its own, simple trajectory information is ambiguous. Such information is not sufficient for the identification of a novel object as animate or inanimate. We turn now to another problem- 
information about the path of motion is not necessary. L. B. Smith, D. Heise, and S. Rivera (in preparation) have shown that 12-month-old infants discriminate a set of displays that are made by pasting together pieces of photographs of animate material from a set of displays that are made by pasting together pieces of inanimate material. By the time that children are 3 years old, they can reason appropriately about unfamiliar animate and inanimate objects presented in photographs. For example, Massey and Gelman (1988) found that 3- and 4-yearold children were able to look at photographs of novel mammalian and nonmammalian animals, statues, wheeled inanimates, and complex rigid inanimates and correctly infer whether each could go both up and down a hill on its own.

Monochrome line drawing reproductions of some of the photographs used by Massey and Gelman are shown in Fig. 6.10. Neither these nor any of the other 14 items could be labelled correctly by separate groups of children of the same age. Despite the evidence that these were unfamiliar objects and despite the fact that they were shown in a still photograph, the children performed very well. They correctly answered that both mammalian and non-mammalian animals could move up and down the hill by themselves, that the wheeled objects could appear to move down but not up the hill by themselves, and that the statues and complex rigid objects could neither go up nor down the hill on their own. It is particularly (p.178) (p.179) interesting that the children did not attribute animacy to the statues or simple machines, even though they were selected to look like familiar animals or to represent objects that sometimes move on their own. The gross shapes of the statues often resembled those of familiar humans or animals more than did the shapes of the unfamiliar animals. Thus the children were already attuned to subtle features of shape and surface character.

Similarly, we see that very young children do not assimilate machines to the animate category. We now return to why we believe that machines are conceived of as a separate hybrid category, i.e. neither animate nor inanimate. 
Massey (1988) provides

additional evidence that shifts

from one conceptual goal to

another are paired with shifts in the kinds of object

characteristics that are treated as relevant. To demonstrate

this, Massey compared the way that groups of 3- and 4-year-old children (as well as adults) organized triads of the MasseyGelman photographs in two different task conditions. In one task the children were asked to pick the two pictures that looked most alike; in the other, the same children were told to pick the two that could go up a hill by themselves. The particular Massey-Gelman stimuli for this study were chosen on the basis of a sample of adults' perceptual similarity ratings of them. Given the ratings, Massey could select appropriate experimental and control triads, i.e. triads that contained pairs that looked alike but crossed the animateinanimate classification (e.g. the echidna and statue shown in Fig. 6.10). Massey assumed that children would choose these pairs during the look-alike task,

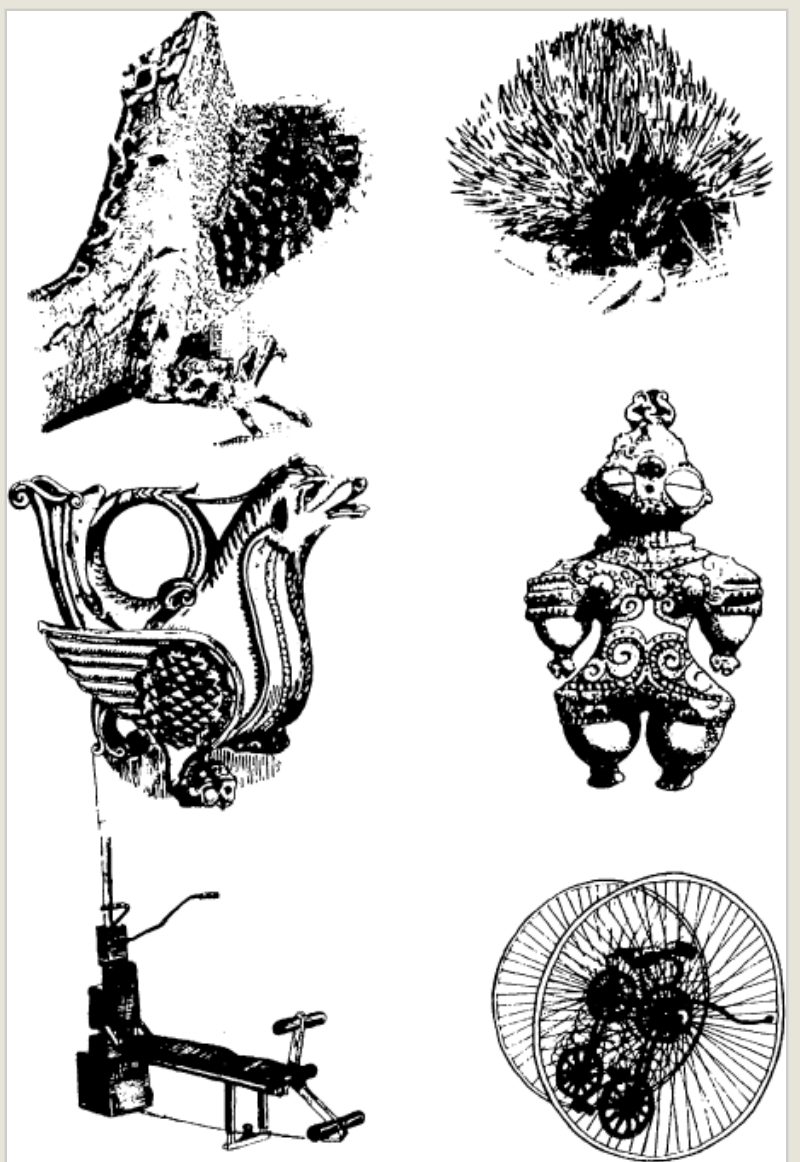

Fig. 6.10 . Line drawings of some of the photographed objects used by Massey and Gelman (1988). Starting on the top row and going from left to right, the samples are a displaying lizard, an echidna, a vessel made to look like a mythical creature, an insect-eyed figurine, an exercise device, and an oldfashioned two-wheeled bicycle. Massey and Gelman did not use line drawings in their study. but not during the up-hill-downhill task.

When analyzing her data, Massey asked whether the children's choices from the look-alike task could predict the answers on the causal task better than an animate-inanimate rule on the causal task. She reasoned that if the up-hill choices were based on judgements of perceptual similarity, as opposed to considerations of an object's animate status, children should select the two items that looked most alike even when deciding which could (or could not) go 
up the hill on its own. This gave her a principled way of comparing the same choices in the up-hill task with same choices in the perceptual similarity task.

Since the items within the animate and inanimate categories tended to look more alike than unalike it is not surprising that Massey reported that the perceptual similarity rule had some predictive validity for both adults and children. Despite this, she found that the conceptual animacy model predicted the causal task choices better than did the look-alike model. This was true for all age groups. Therefore surface cues that controlled similarity judgements did not control answers to the causal questions. More importantly for the discussion here, since the stimuli were of static objects, it is clear that animacy decisions can be rendered even when an object is not moving.

(p.180) Other details of the Massey-Gelman study show that the children looked for animate-relevant information in the pictures, even if this meant attending to minute and seemingly non-salient details. For example, they focused on the antennae of a crustacean when explaining why it could move up a hill, and seemed to ignore the fact that it had a large shiny surface. In contrast, the same children stated that statues could not move because they were too shiny! Some children even claimed that an unknown animate object could move itself because it had legs, a rather startling claim for objects like the echidna whose limbs were not visible in the photograph. However, 'limbs' per se did not suffice for a child: statues with limbs were not able to move themselves because they lacked 'real' feet, they were 'furniture animals', 'pretend', or 'markedup' (i.e. patterned on the surface), or they were made of the 'wrong stuff', be the 'stuff metal', wood, or plastic (Gelman 1990).

The children's claims that statues did not have 'real' feet or that animals had limbs when none were visible are consistent with the idea that the information in the pictures was interpreted with reference to causal principles. Metal feet cannot serve as an agent of self-initiated motion, no matter how much they resemble what the children call 'real' feet. However, the capacity for selfinitiated motion is typically realized through limb action, so why not assume that these are part of the animal in the picture, whether or not they are shown?

Kremer (1989) confirms the early salience of subtle cues in photographs for motion-pose and type of material. When shown, one at a time, a series of pairs of photographs of live animals and quality three-dimensional copies (Lenox porcelains), 4-year-old children readily identify each as 'real' or 'fake'. A similar trend for 3-year-old children was mitigated by their problems with the ambiguous terms real, fake and pretend. In an ongoing study by Gelman and Meck that is designed to minimize such ambiguity, we are finding that even 3year-old children can systematically distinguish between a photograph of a real and a fake (or pretend) animal. 
How should we account for the above developmental findings? We explicitly exclude accounts that equate this early knowledge with innate knowledge. At least in this case, this theoretical move would amount to saying that we are born with an infinitely large list of innate perceptual detectors. Our preference is to postulate a powerful innate engine of learning. The idea is that we benefit from innate first principles that define the domain and drive the learning in the domain. These principles, although skeletal in form, are at least as much conceptual as perceptual. From the outset, the system gathers data that are relevant to pivotal conceptual issues and/or distinctions to which no single class of perceptual factors is an unfailing guide. In the animate-inanimate case these conceptual issues often involve the causes of motion, but they are not restricted to them. Causal (p.181) principles are often concerned as much with the composition and characteristics of the objects as the energy conditions that underlie their movements.

It follows that our account of the developmental data is that the young children's causal principles serve to encourage attention to, and storage of, relevant information about animate and inanimate objects. Conceptual concerns allow them to reach the point where they already know enough about the kinds of data that tend, on average, to characterize animate and inanimate objects. When they are faced with novel examples, they know enough to make surprisingly accurate guesses on the basis of relevant perceptual features. However, it would be a mistake to conclude from such abilities that perceptual features alone are driving the identification and inference processes. If this were so, the children should not have constructed non-visible elements when looking at photographs of animals, and they should have mistakenly treated statues as if they were animate things. Further, they should not have been able to distinguish photographs of 'real' animals from photographs of porcelain reproductions.

\section{Summary}

Motion is no less ambiguous or indeterminative of ontological category than are static features of objects. Young and mature alike must use additional resources to achieve correct identification of novel cases of animate and inanimate objects and events. A principled concern with the causes, or sources of the movements of separately moveable objects, encourages us to treat as relevant those attributes and conditions that characterize animate and inanimate objects and their kinds of movements. In this way our conceptions and perceptions of animate and inanimate objects work together. The more that the input supports the related conceptual structure, the greater is the probability that the attribution will be consistent with the input. However, when data are ambiguous or when there are gaps in the data, we can count on our conceptual structure to assist us. 
Machines are a particularly interesting category. Although they appear to move on their own, they are quintessentially ambiguous. They are made of inanimate material and do not exhibit biomechanical motions, nor do they adjust very well to local environmental problems. Robots are not particularly good at adjusting their motions to local perturbations in the environment; in contrast, so predictable is the animate world's ability to deal with unanticipated holes, bodies of water, oil slicks, branches that come below the head, sun in the eyes, weather changes, etc., that we almost forget how remarkable are the action abilities of the animate world. This reflects the fact that machines do not exhibit the kind of action that is controlled by biological mechanisms (e.g. coupled oscillators) - action (p.182) patterns that are noted by infants (Berthental 1993) and used as cues for animacy (Wilson 1986). Additionally, machines are made of the wrong 'stuff to be treated as animate. Recall that causal principles yoke information about objects' trajectories and their kind of 'stuff'. If so, machines cannot be classified as examples of one or the other ontological category. Such considerations led us to expect that children and adults would create a new hybrid category for representing machines. All the data presented in this chapter are consistent with this expectation. This is yet another reason to conclude that we cannot assign a novel object to either the animate or inanimate category simply on the basis of information about whether we see that it moves on its own or not.

We have challenged bottom-up models on the grounds that we believe that there is no list of perceptually accessible features that will always tell us what is animate and what is inanimate. The perceptual information, as informative as it might be, is nevertheless usually ambiguous or incomplete. What determines one's categorization of an object is, in the end, conceptual. We have presented data to show that this is true for both adults and young children. Ambiguity is resolved with respect to choices of causes about objects and their motions, given a set of conditions. If, during a trip to the desert, you came upon a round object covered with needle-like protrusions, you probably would call it a 'cactus'. However, should it start to move, you would surely be relieved to find out that it was a non-dangerous echidna and would not insist that it was a cactus because of what it looked like initially. Like our subjects, you might even start to look for its limbs.

\section{Acknowledgements}

Partial support for the preparation of this chapter as well as some of the research reported here came from grants from the National Science Foundation (BNS 89-16220 and DBS-9209741) and a University of California at Los Angeles (UCLS) Dean's fund to Rochel Gelman. Analyses of the Stewart data and the follow through experiments were accomplished with funds from the same sources as well as graduate student support from the Universities of Pennsylvania and Virginia to Frank Durgin. We gratefully acknowledge the many people who helped us think through and/or code the event analyses of the 
moving sprite experiments, especially Lisa Becker, Randy Gallistel, Nancy Henley, Betty Meck, and Andy Su. Last and most importantly, we are indebted to Judith Stewart who shared so much with us. We are grateful to Mary McManus for the freehand line drawings in Fig. 6.10.

\section{(p.183) References}

Bibliography references:

Armstrong, S. L., Gleitman, L. R. and Gleitman, H. (1983). What some concepts might not be. Cognition 13, 263-308.

Baillargeon, R. (1987). Young infants' reasoning about the physical and spatial properties of a hidden object. Cognitive Development 2, 179-200.

Baillargeon, R., Spelke, E. S. and Wasserman, S. (1985). Object permanence in 5month-old infants. Cognition 20, 191-208.

Bassili, J. N. (1976). Temporal and spatial contingencies in the perception of social events. Journal of Personality and Social Psychology 33, 680-5.

Beedham, C. (1987). The English passive as an aspect. Word 38, 1-12.

Berthental, B. I. (1993). Infants' perception of biomechanical motions: instrinsicimage and knowledge-based constraints. In Carnegie Symposium on cognition. Visual perception and cognition in infancy (ed. C. Granrud). Erlbaum, Hillsdale, NJ.

Carey, S. (1985). Conceptual change in childhood. Cambridge University Press.

Cochran, W. G. and Cox, G. M. (1957). Experimental Designs. Wiley, New York.

Gallistel, C. R. (1980). From muscles to motivation. American Scientist 68, 398409.

Gelman, R. (1990). First principles organize attention to and learning about relevant data: number and the animate-inanimate distinction as examples. Cognitive Science 14, 79-106.

Gelman, R. and Brenneman, K. (1993). First principles support universal and culture specific learning about numbers and music. In Mapping the mind domains, culture and cognition (ed. L. Hirschfeld and S. Gelman). Cambridge University Press.

Gleitman, L. R. (1990). The structural source of verb meaning. Language Acquisition 1, 3-55.

Goffman, E. (1974). Frame analysis. Harvard University Press, Cambridge, MA. 
Distinguishing between animates and inanimates: not by motion alone

Golinkoff, R. M. and Harding, C. G. (1980). Infants' expectations of the movement potential of inanimate objects. In International Conference on Infant Studies, New Haven, CT.

Heider, F. and Simmel, M. (1944). An experimental study of apparent behavior. American Journal of Psychology 57, 243-59.

Hochberg, J. (1978). Perception (3rd edn). Prentice-Hall, Englewood Cliffs, NJ.

Kellman, P. and Spelke, E. (1983). Perception of partly occluded objects in infancy. Cognitive Psychology 15, 483-524.

Kremer, K. (1989). Preschoolers can distinguish between representations of fake and real animals. Unpublished Master's Thesis, University of California at Los Angeles.

Lamb, S. (1991). Acts without agents: an analysis of linguistic avoidance in journal articles on men who batter women. American Journal of Orthopsychiatry 61, 250-7.

Leslie, A. and Keeble, S. (1987). Do six-month-olds perceive causality. Cognition $25,265-88$.

Mandler, J. M. (1992). How to build a baby II. Conceptual primitives.

Psychological Review 99, 587-604.

Massey, C. (1988). The development of the animate-inanimate distinction in preschoolers. Unpublished PhD Thesis, University of Pennsylvania.

(p.184) Massey, C. and Gelman, R. (1988). Preschoolers decide whether pictured unfamiliar objects can move themselves. Developmental Psychology 24, 307-17.

Michotte, A. (1963). The perception of causality. Methuen, London.

Murphy, G. L. and Medin, D. L. (1985). The role of theories in conceptual coherence. Psychological Review 92, 289-316.

Pinker, S. (1989). The learnability of argument structure. MIT Press, Cambridge, MA.

Poulin-Dubois, D. and Schultz, T. R. (1988). The development of the understanding of human behavior: from agency to intentionality. Developing theories of mind (ed. J. W. Astington, P. L. Harris, and S. Warren) Cambridge University Press, Cambridge. 
Distinguishing between animates and inanimates: not by motion alone

Smith, L. B., Heise, D. and Rivera, S. (in preparation). Surface gradients in 12month-olds' discrimination of animals versus vehicles. Unpublished MS, Indiana University, Bloomington, IN.

Stanley, J. P. (1975). Passive motivation. Foundations of Language 13, 25-39.

Stewart, J. A. (1982). Perception of animacy. Unpublished Dissertation, University of Pennsylvania.

Stewart, J. (1984). Object motion and the perception of animacy. Presented at the meeting of the Psychonomic Society, San Antonio, TX, November 1984.

Weiner, E. J. and Labov, W. (1983). Constraints on the agentless passive. Journal of Linguistics 19, 29-58.

Wilson, N. J. (1986). An implementation and perceptual test of a principled model of biological motion. Unpublished masters' thesis, University of Pennsylvania.

Notes:

(*) We thank Judith Stewart for providing us with such full access to her unpublished data and writings.

(*) Our displays were produced and displayed on a Macintosh SE with a frameby-frame sprite program Videoworks II ${ }^{\circledR}$ synchronized with the screen refresh using a Videoworks Accelerator ${ }^{\circledR}$ and presented by means of a HyperCard ${ }^{\circledR}$ interface. 